\title{
RENDIMIENTO ACADÉMICO OBTENIDO CON UN APLICATIVO MULTIMEDIAL VS CLASE TRADICIONAL
}

\section{ACADEMIC PERFORMANCE ACHIEVED WITH A TRADICIONAL CLASS VS MULTIMEDIAL APLICATION}

Celio E. Pineda Rodríguez ${ }^{1}$

\section{RESUMEN}

El propósito de este estudio fue analizar los efectos sobre el aprendizaje, utilizando el recurso multimedial "Autoaprendizaje de la Biología General", en el curso académico Ciencia Biológicas I, conformado por treinta estudiantes de la facultad de Medicina Veterinaria de la Universidad de Ciencias Aplicadas y Ambientales, U.D.C.A. En principio, el proyecto se fundamentó en las teorías de aprendizaje que han influido en el diseño de aplicaciones orientadas a la adquisición de conocimiento. Como el grupo no se podía aleatorizar en su conformación, se utilizó un diseño cuasiexperimental, para lo cual se dividió en dos subgrupos y, alternadamente, se aplicó el recurso multimedial, de manera que el subgrupo sirviera de control, caso en el que recibió la temática correspondiente, mediante una clase tradicional. Se analizaron las diferencias de rendimiento académico entre cada subgrupo y entre los subgrupos; en este caso, el de control que utilizó la clase tradicional y para este tipo de tratamiento, se empleó el diseño cruzado o crossover. Se encontró que el rendimiento académico, en temas como las proteínas y los lípidos, la aplicación tiene efectos positivos, mientras que en otros no hay diferencia entre los dos recursos. En cuanto al diseño de las actividades del aplicativo, no es clara la relación con el rendimiento, ya que cuando

${ }^{1}$ Ingeniero de Alimentos, Esp. Ingeniería de la Calidad, Magister en Docencia. Universidad de Ciencias Aplicadas y Ambientales U.D.C.A. Cra 102 No. 83-96 Int. 1 Apto. 403. cpinedar@udca.edu.co estan presentes las animaciones, el rendimiento es más bajo y pareciera que, con las actividades de texto fijo e imágenes, es más alto.

Palabras clave: Multimedia, rendimiento, TIC, aprendizaje autónomo.

\section{SUMMARY}

The purpose of this research was to analyze the effects on learning, when using the multimedia resource "Autolearning Biology" in the academic course Biological Science I, composed of 30 students of Veterinary Medicine at the University of Applied Sciences and Environmental UDCA. Initially, the project was based on the learning theories which influenced the design of applications, aimed at the acquisition of knowledge. As the group could not be aleotorized, we used a quasiexperimental design, divided into two subgroups. The multimedia resource was alternately applied in a way that a subgroup could serve as control, in which case the thematic received, corresponded to traditional classroom teaching. Differences among the academic performance of each subgroup and among subgroups were established, being the control the traditional classroom teaching. For this type of treatment a crossover design was used. It was found that for the academic performance in areas such as proteins and lipids, the application of the multimedia resource had a positive impact on performance, while in other areas no difference between the two applications was obtained. As for the design of the activities of application, no clear connection with the performance was detected, since 
when animations were used, the yield was lower and apparently with the activities of fixed text and images, the performance is higher.

Key words: Multimedia learning, TIC, autonomous learning.

\section{INTRODUCCIÓN}

Con el cambio de siglo, también vino el cambio de sociedad, hoy llamada "sociedad de la información" (Cabero et al. 2003). Para Rodríguez \& Planchuelo (2005), en esta nueva etapa de la historia de la humanidad, denominada por otros como "sociedad del conocimiento" o "era de la información", la Internet, las TIC (Tecnologías de la Información y la Comunicación) y la información serán las que impulsen y protagonicen el desarrollo social, científico, económico, cultural y educativo que caracterice el nuevo modelo de sociedad. En el campo educativo, algunos consideran que la institución escolar debe formar a personas competentes, críticas y con capacidad de trabajo autónomo (Presidencia de la República, 2003) y cooperativo, interviniendo en dos grandes campos: el uso reflexivo de la tecnología y el desarrollo de las habilidades de la información. Estos cambios, según los describe Alba (1998), han permitido que muchas personas tengan las mismas facilidades de acceso a la información (bibliotecas virtuales) o a la formación (discapacitados) o que personas que por sus limitaciones era imposible o poco factible que pudieran trabajar, con ello tengan ahora opción en el mundo laboral.

En la educación superior, según Bartolomé (1994), los tutoriales son los más utilizados en "Educación Superior, aunque su fundamentación en una teoría asociacinista del aprendizaje los hace poco atractivos hoy en día. El modelo es adecuado para la adquisición de conocimientos, especialmente en lo que se refiere a aspectos relacionados con la retención de información". El mismo autor cree que los docentes pueden ser los creadores de los materiales orientados al aprendizaje.

En contraposición el uso de medios alternativos en la educación ha existido la clase tradicional en la que los temas son presentados de lo particular a lo general, con énfasis en conceptos básicos. Se enfatiza en que los alumnos sigan al pie de la letra lo presentado en el currículo y las actividades se basen en la información de los libros de texto. Los estudiantes son vistos como "tabulas rasas", tableros en blanco, donde los docentes depositan toda la información. Éstos transmiten el conocimiento a los alumnos, que sólo son receptores y esperan escuchar la respuesta correcta para así validar el aprendizaje. La evaluación, se considera como algo separado de la enseñanza y ocurre, generalmente, a través de las pruebas.

Dentro de las funciones sustantivas de la educación superior está la docencia y, junto con ella, la producción académica de los docentes, de la cual forma parte la creación de materiales educativos orientados a complementar la formación y mejorar el rendimiento académico de los alumnos. Es posible validar y ajustar recursos multimediales orientados al aprendizaje.

La importancia de este estudio también radica en ayudar a establecer qué está pasando en la formación universitaria con el empleo de las TIC, con las cuales se han elaborado numerosos recursos multimediales, que se están incorporando en la enseñanza. En la actualidad, una significativa parte de ellos, se halla en Internet y se ha constituido en apoyo decisivo para los docentes y los estudiantes de los distintos niveles de la educación, tanto de tipo presencial como a distancia.

Lo anterior concuerda, con las circunstancias actuales en materia de políticas educativas del Estado colombiano, Presidencia de la República (2003), con relación al aprendizaje autónomo. Esto, se pone de relieve en la publicación del Consejo Nacional de Acreditación CNA (2003) que hace referencia, en esta misma dirección, mencionando que cada día se hace más necesario contar con materiales que le permitan al estudiante el aprendizaje autónomo, como uno de los indicadores de la acreditación de programas de pre-grado. Uno de tales materiales es el recurso multimedial, que puede ofrecer ventajas significativas, al menos en el campo técnico. Como sus efectos en los estudiantes no son claros aún, la presente propuesta apunta a dilucidar tales alcances, pues el conocimiento generado puede abrir posibilidades en el diseño y en el empleo de los materiales en mención.

Para intentar resolver lo expuesto, se planteó determinar el efecto del aplicativo multimedial "Autoaprendizaje de la Biología General" sobre el rendimiento, en estudiantes de primer semestre, que cursan la asignatura Ciencias Biológicas I, de la facultad de Medicina Veterinaria, en la 
Universidad de Ciencias Aplicadas y Ambientales U.D.C.A. En el entendido por Edel (2003), rendimiento académico es el nivel de conocimientos demostrado en un área o asignatura, comparado con la norma; desde la perspectiva de otros, la complejidad del rendimiento académico va desde la misma conceptualización; según este autor, en ocasiones se le denomina aptitud escolar, otras como desempeño académico, aunque en la vida escolar son sinónimos. Para Himmel (1985), en Andrade et al. (2001), el rendimiento escolar o efectividad es definido como nivel de logro de los objetivos establecidos en los programas académicos. En cuanto a la relación del tipo de enseñanza y la variable rendimiento obtenidos en educación a distancia, en modalidad e-learning, resultó en la falta de relación entre una y otra (Recio \& Cabero, 2005).

Los recursos utilizados en aprendizaje presentan resultados diversos, con lo cual no es posible predecir sus efectos, aun cumpliendo con aspectos de diseño y metodología de enseñanza; la temática es crucial a la hora de validar estos recursos.

\section{MATERIALES Y MÉTODOS}

Se utilizó el método cuasi-experimental donde se manipula intencionalmente, al menos una variable independiente, para ver su efecto y relación con una o más variables dependientes, tal como lo expresa Hernández et al. (1997): en estos diseños cuasi-experimentales, los miembros no son asignados al azar a los grupos, ni emparejados, sino que dichos grupos ya estaban formados antes del experimento, son grupos intactos (la razón por la que surgen y la manera como se formaron fueron independientes o aparte del experimento).

Debido al tamaño del grupo y para aumentar la confiabilidad, se utilizó el diseño cross over o alternativo, también conocido como diseño conmutativo o cruzado. Es un plan de investigación cuasiexperimental en el que cada sujeto recibe una secuencia de tratamientos en un orden determinado, por la estructura del propio diseño, con el claro objetivo de estudiar diferencias entre los tratamientos individuales. Se debe señalar que en este diseño el grupo es control de sí mismo. La comparación se realiza utilizando una estrategia intrasujeto, de forma que cada participante actúa como su propio control.

Para el estudio, se tuvo en cuenta un grupo existente, al que se le considera población y de tamaño treinta, que es el número máximo que, de acuerdo con el proyecto educativo institucional de la U.D.C.A, puede tener un grupo; los alumnos oscilaban entre 17 y 20 años. Para el estudio, la mayoría de la población eran mujeres, en el subgrupo $A, 60 \%$ y en $B, 67 \%$. El único criterio excluyente es la repitencia del curso, estos estudiantes participan de la investigación, pero sus resultados no son tenidos en cuenta. En estudios de este tipo, la muestra se puede dividir sin que sea necesaria la igualdad entre los subgrupos (Sanhueza-Tsutsumi et al. 2005).

El experimento fue realizado en un aula y en una sala de informática de la Universidad, en la que se impartió la clase, utilizando el aplicativo multimedial "Autoprendizaje de la Biología General”. Para obtener información de los aspectos abordados por la investigación, se utilizaron distintas formas de recopilación. Para determinar el comportamiento de los alumnos frente al empleo de la Aplicación Multimedial, el instrumento contó con un registro del tiempo y frecuencias de uso, entre otros ítems. Para el rendimiento, el profesor realizó pruebas, las mismas para los dos subgrupos.

Los rendimientos obtenidos por cada alumno, por subgrupo, fueron comparados utilizando la prueba t, por medio del software Graph Pad. El estadístico t: o valor de contraste, con el que se obtiene la probabilidad de ocurrencia p, que puede ser analizada con una cola, la de la izquierda, si se espera que los valores obtenidos en el tratamiento ocurran con mayor frecuencia en esta zona y viceversa. Para el caso de este estudio, como los valores del rendimiento pueden estar en cualquiera de las dos zonas, el análisis se ha hecho teniendo en cuenta dos colas. El valor-p, que usualmente reportan la mayoría de paquetes estadísticos, es la probabilidad de obtener, según esa distribución, un dato más extremo que el que proporciona el test; refleja también la probabilidad de obtener los datos observados si fuese cierta la hipótesis inicial. Si el valor-p es muy pequeño (usualmente se considera $p<0.05$ ), es poco probable que se cumpla la hipótesis de partida y se debería rechazar. La región de aceptación corresponde, por lo tanto, a los valores centrales de la distribución para aquellos de p>0.05.

\section{RESULTADOS Y DISCUSIÓN}

Rendimiento académico del subgrupo A (Figura 1): comparados los rendimientos obtenidos por los integrantes del subgrupo A en el tema lípidos y carbohidratos, la diferencia estadística (p para dos colas 
$=0,0167)$ muestra que el aprendizaje mediado por una clase tradicional es más efectivo que la aplicación; este comportamiento, se puede deber al cambio de metodología.

En los temas lípidos y proteínas no hay diferencia en el rendimiento académico; sin importar la metodología utilizada los estudiantes aprueban. Así mismo, no hay diferencia estadísticamente significativa (valor $\mathrm{p}$ para dos colas $=0,8757$ ), resultado similar al hallado por Marrison E Frick (1993)

Cuando los estudiantes abordaron el tema enzimas, su rendimiento fue inferior al mínimo aprobatorio, sugiriendo que es un tema de difícil aprendizaje. Comparándolo con el de proteínas en el que se usó el aplicativo, no presenta una diferencia significativa (valor p para dos colas: 0,1032 ).

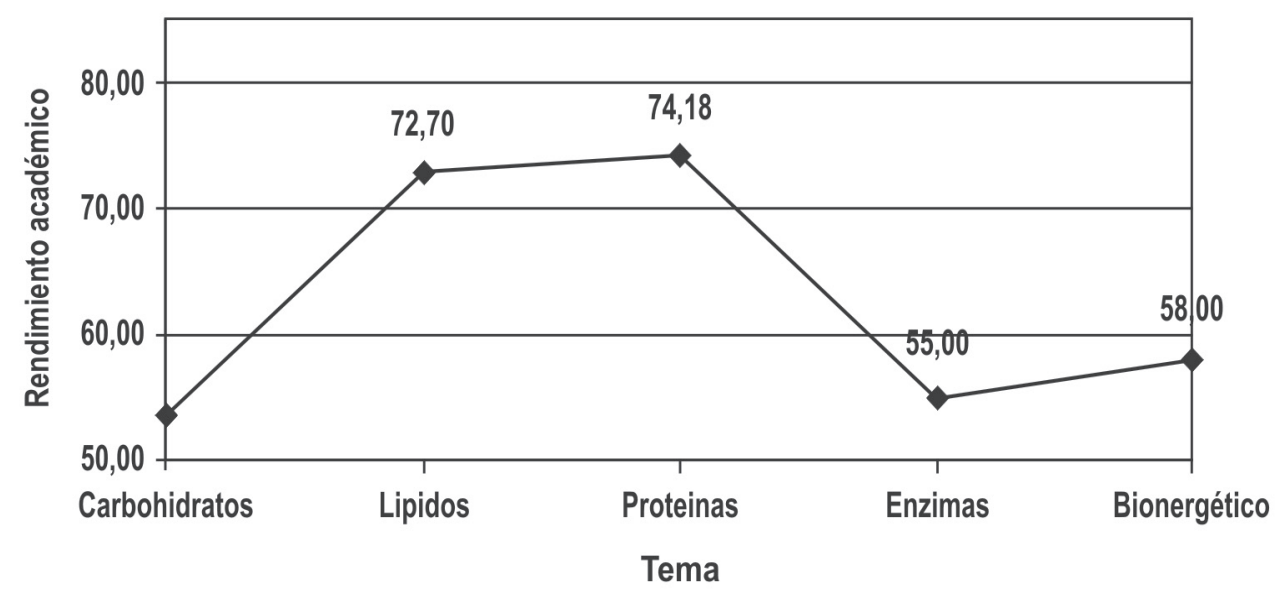

Figura 1. Rendimiento académico subgrupo $A$, utilizando alternadamente aplicación multimedial, tratamiento (T) y clase tradicional, control (C).

Con relación al aprendizaje del tema bioenergética abordado con la aplicación, los estudiantes no logran el aprendizaje; en este tema parece que la intervención del docente es necesaria para su comprensión, comparado con la clase tradicional; tema enzimas, no hay diferencia significativa (valor p para dos colas $=0,7702$ ).

En el subgrupo A también se analizó el rendimiento académico global al finalizar el experimento, esto es, el rendimiento obtenido mediante la clase tradicional y el obtenido utilizando la aplicación.

Al analizar globalmente el rendimiento académico del subgrupo A, este fue muy similar tanto con clase tradicional como con el aplicativo. Cualquiera que sea la metodología aprueban los temas abordados.

Rendimiento académico del subgrupo B: el subgrupo $\mathrm{B}$, se analizó de manera idéntica. Como primer tema, se abordaron los carbohidratos, mediante la clase tradicional (C) y, luego, se utilizó la aplicación tratamiento $(\mathrm{T})$, tal como se muestra en la figura 2.
Este grupo recibió el tema carbohidratos, inicialmente, mediado por la clase tradicional, aprobándolo y el tema lípidos, mediado por el recurso multimedial, observándose que no importa la metodología utilizada, los estudiante logran un aprendizaje aprobatorio (valor $\mathrm{p}$ para dos colas $=0,3186$ ). En estudios en el campo de la educación básica en ciencias, se concluye que existe un incremento notorio en el rendimiento académico de los estudiantes cuando utilizan recursos multimediales. Uno de estas investigaciones (Moënne et al. 2004), concluye que hay "un claro y medible incremento de los aprendizajes en los alumnos que usaron metodologías apoyadas con la multimedia".

Al utilizar el aplicativo para el aprendizaje de las proteínas, se encuentra que los estudiantes no logran aprobar el tema, si se compara con el anterior tomado con el profesor; se advierte, por la altamente significancia (valor $\mathrm{p}$ para dos colas $=0,0010$ ), que es un tema que demanda la presencia del docente, con lo que queda evidente que la aplicación no produce los efectos esperados, al menos para este subgrupo o que podría ser un complemento, 


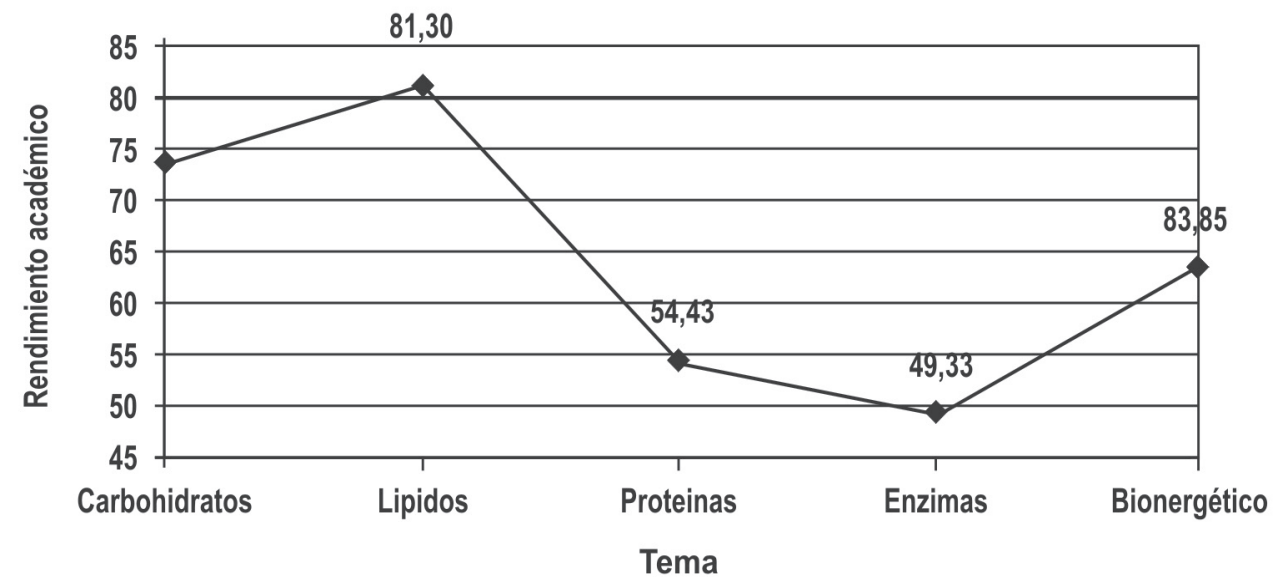

Figura 2. Rendimiento académico sugrupo B, utilizando alternandamente apllcación multimedial y clase tradicional.

como lo reporta Healy et al. (2005), en el que la experiencia ha sido que esa utilización de los estudiantes del programa no se asoció a mejoras del grupo.

La comparación de los dos últimos temas evidencia la dificultad de éstos, porque con ninguna de la dos metodologías logran los mínimos aprobatorios, sugiriendo que sin importar el método empleado, el rendimiento académico en estos temas, sería bajo.

Con el tema bioenergética, los estudiante logran superar los mínimos necesarios para aprobar; sin embargo, al comparar los rendimientos de bionergética con enzimas no hay diferencia significativa (valor p para dos colas $=0,0863$ ), considerando que en el tema enzimas, no aprobaron.
Al analizar el rendimiento global hay una ligera diferencia a favor de la aplicación. Esta diferencia no es significativa, lo cual sugiere que para las temáticas tratadas, se consigue una aprobación independiente del recurso de aprendizaje que utilicen.

Rendimiento académico de dos subgrupos en el mismo tema: los dos subgrupos iniciaron simultáneamente: A, con la aplicación y B, con la clase tradicional. Esto permitió que se pudiera hacer la comparación en el mismo tema, donde uno de ellos hace las veces de control y el otro de grupo experimental, tal como se muestra en la Tabla 1.

Los resultados de los subgrupos en el tema carbohidratos, la aplicación no mejora el rendimiento

Tabla 1. Resultados de la aplicación de la prueba t-student al rendimiento obtenido por los dos subgrupos en el mismo tema.

\begin{tabular}{|c|c|c|c|c|c|c|c|c|c|c|}
\hline & \multicolumn{2}{|c|}{ Carbohidratos } & \multicolumn{2}{|c|}{ Lípidos } & \multicolumn{2}{|c|}{ Proteínas } & \multicolumn{2}{|c|}{ Enzimas } & \multicolumn{2}{|c|}{ Bioenergética } \\
\hline & $\begin{array}{c}\mathrm{T} \\
\text { (Subgrupo } \\
\text { A) } \\
\end{array}$ & \begin{tabular}{|c|} 
C \\
(Subgrupo \\
B) \\
\end{tabular} & \begin{tabular}{|c|} 
C \\
Subgrupo \\
A) \\
\end{tabular} & \begin{tabular}{|c|}
$\mathrm{T}$ \\
(Subgrupo \\
$\mathrm{B})$ \\
\end{tabular} & $\begin{array}{c}\mathrm{T} \\
\text { Subgrupo } \\
\mathrm{A}) \\
\end{array}$ & $\begin{array}{c}\text { C } \\
\text { Subgrupo } \\
\text { B) }\end{array}$ & $\begin{array}{c}\mathrm{T} \\
\text { Subgrupo } \\
\mathrm{B}) \\
\end{array}$ & $\begin{array}{c}\text { C } \\
\text { Subgrupo } \\
\text { A) }\end{array}$ & $\begin{array}{c}\text { C } \\
\text { Subgrupo } \\
\text { B) } \\
\end{array}$ & $\begin{array}{c}\mathrm{T} \\
\text { Subgrupo } \\
\text { A) }\end{array}$ \\
\hline Media & 53,64 & 74,17 & 72,7 & 81,3 & 74,18 & 54,43 & 55,00 & 49,33 & 58,00 & 63,85 \\
\hline Observaciones & 11,00 & 12,00 & 11,00 & 15,00 & 11,00 & 14,00 & 10,00 & 15,00 & 10,00 & 13,00 \\
\hline $\begin{array}{l}\text { Diferencia hipotética } \\
\text { de las medias }\end{array}$ & 0,00 & & 0,00 & & 0,00 & & 0,00 & & 0,00 & \\
\hline Grados de libertad & 21,00 & & 24,00 & & 23,00 & & 23,00 & & 21,00 & \\
\hline Estadístico t & 2,67 & & 1,2702 & & 2,09 & & 0,6041 & & 0,6689 & \\
\hline Desv. Est. & 20,14 & 16,74 & 13,48 & 19,22 & 27,29 & 20,01 & 23,69 & 22,51 & 21,50 & 20,22 \\
\hline Valor $p$ dos colas & 0,0145 & & 0,2162 & & 0,0478 & & 0,5517 & & 0,5109 & \\
\hline Error Est. de la media & 6,07 & 4,84 & 4,07 & 4,96 & 6,23 & 5,35 & 7,49 & 5,81 & 6,8 & 5,61 \\
\hline
\end{tabular}


y en comparación con la clase tradicional hay una diferencia estadísticamente significativa ( $p=0,0145)$, en contra del grupo experimental. Parece ser que el grupo que recibe el tratamiento es afectado negativamente al abordar el tema con esta nueva didáctica (aplicativo).

En el tema lípidos, el aplicativo logra un mejor rendimiento que el grupo control, aunque no hay no hay diferencias significativas.

Al analizar el tema proteínas, se observa nuevamente que el aplicativo tiene un efecto positivo sobre los estudiantes que lo utilizaron y que la diferencia se considera significativa ( $\mathrm{p}$ $=0,0478)$, al compararlo con el rendimiento obtenido en el mismo tema, pero con la clase tradicional

En el tema enzimas, además de no existir diferencia significativa ( $p$ dos colas $=0,517$ ), se encuentra una particularidad: ninguno de los subgrupos, al menos en promedio, aprueba (rendimiento menor a 60 ), aunque se observa un mejor comportamiento de la media en la clase tradicional. Esto sugiere que, sin importar la metodología utilizada, los rendimientos van a ser bajos al parecer por la dificultad del mismo tema.

En el último tema analizado, bioenergética, no existe diferencia significativa ( $p=0,5109)$; sin embargo, los estudiantes aprenden más con la presencia del docente, es decir, en la clase tradicional.
En este sentido, los resultados de la investigación concuerdan con estudios en los cuales se han utilizado aplicativos multimediales versus la clase tradicional, para comparar el rendimiento académico de los estudiantes, como el de Smith et al. (2006); el análisis de los resultados mostró que no había diferencias significativas entre los resultados de la clase tradicional frente a los obtenidos con el uso de la herramienta multimedial. Igual comportamiento encontró Lernihan (2002).

Rendimiento académico de los dos subgrupos al final de la aplicación (Figura 3): en este caso interesaba saber qué efecto habían producido tanto el aplicativo como la clase tradicional sobre el rendimiento académico, teniendo en cuenta los rendimientos del subgrupo A y del $\mathrm{B}$. El subgrupo A recibió el tratamiento tres veces y el $\mathrm{B}$, dos. Con la clase tradicional ocurrió lo contrario: $\mathrm{A}$, dos veces y $B$, tres veces. Teniendo en cuenta los rendimientos promedio de ambos subgrupos expuestos a la aplicación y a la clase tradicional, se analizó como si fuera un solo grupo y se puede decir que el rendimiento académico del grupo no se ve afectado negativamente por la metodología propuesta, ya que obtienen rendimientos similares; se encuentra que los estudiantes aprenden lo mismo con cualquiera de las dos metodologías aplicadas, la clase tradicional o el uso de la aplicación multimedial, lo cual concuerda con el primer tipo de trabajos mencionados antes.

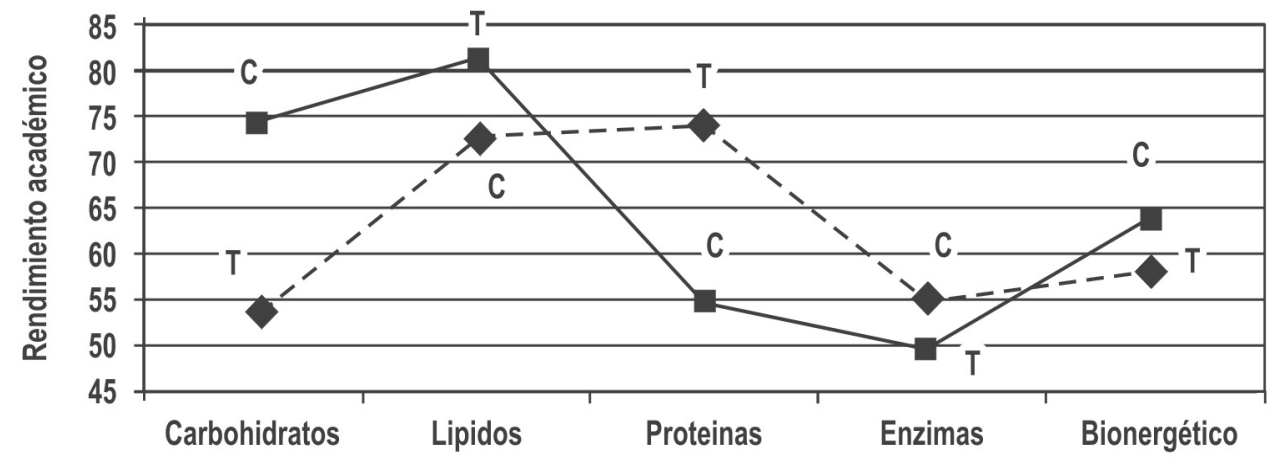

-マ-Subgrupo A $\rightarrow$ Subgrupo B

Figura 3. Comparación del rendimiento académico de los estudiantes de los dos subgrupos, utilizando alternadamente la aplicación y la clase tradicional. 
Se llega a la misma conclusión en otro estudio y llevado a cabo en la Pontificia Universidad Javeriana, con estudiantes de medicina, en el aprendizaje de la farmacología, donde se recibieron las clases utilizando los medios TIC y la clase tradicional. Este trabajo fue realizado por Castaño et al. (2001), quienes concluyeron que "el promedio de notas obtenido por los estudiantes del grupo estudio fue similar al de los estudiantes del grupo control, lo cual hace suponer que el efecto final de la calificación no se vería modificado con la modalidad de enseñanza a la que se expongan los estudiantes". Lo mismo obtuvieron en "Computer multimedia instruction versus traditional instruction in post-secondary agricultural education" (Marrison $\mathcal{E}$ Frick, 1993).

Al hacer una análisis descriptivo de los rendimientos obtenidos por los alumnos teniendo en cuenta el género, se encontró que, independiente del recurso utilizado la mujeres siempreaprueban lostemas, tal como semuestra en lafigura 4 , no se hacen inferencias por la reducción de la muestra al estratificar los subgrupos.
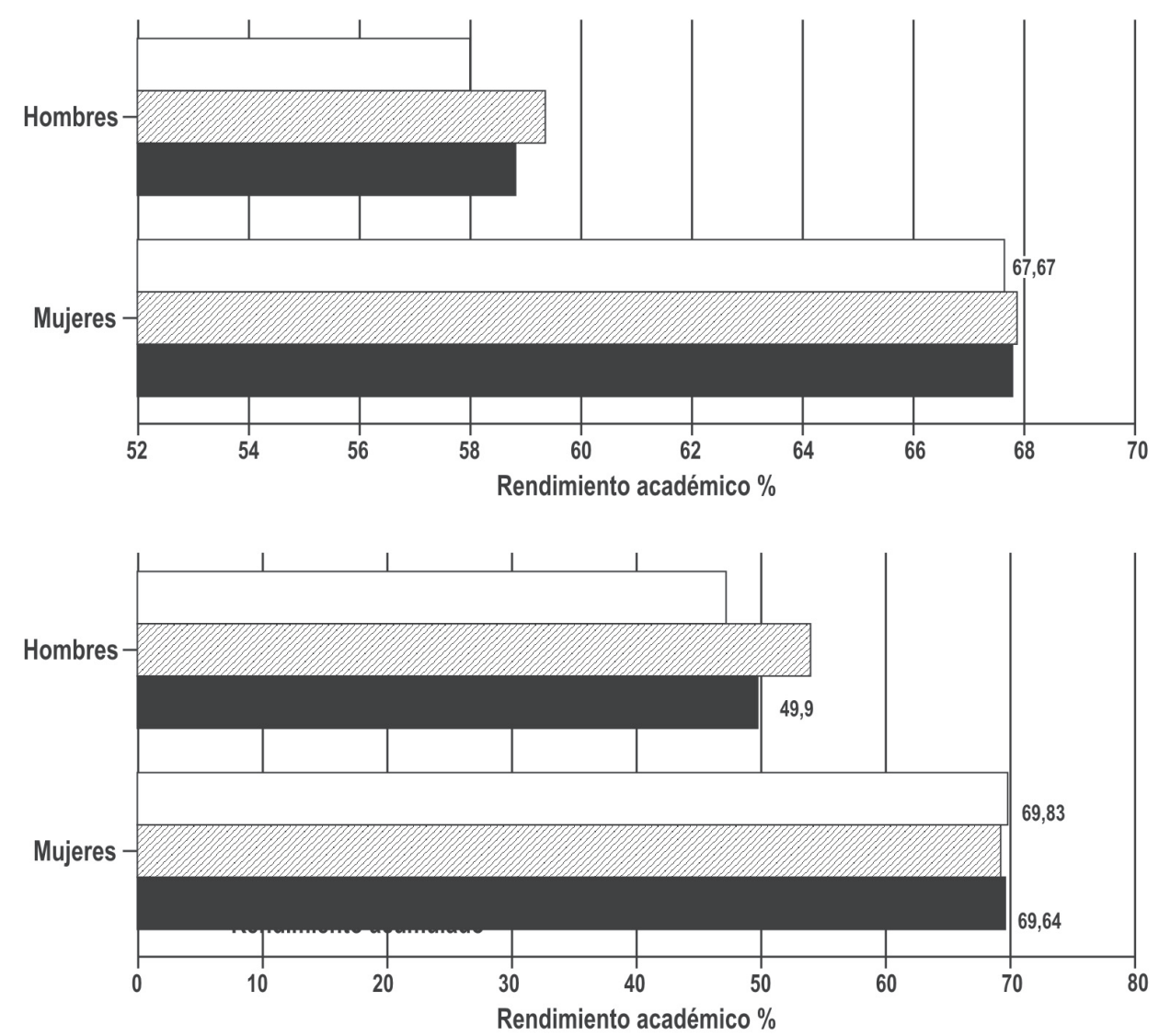

Figura 4. Rendimiento académico por género.

Los temas tratados en el curso tienen distintos niveles de dificultad, por lo cual es entendible que algunos requieran de un mayor apoyo del docente que otros. Este parece ser el caso de los temas enzimas y bioenergética, en donde los resultados favorecen el aprendizaje mediante una clase tradicional.
Enlos casos cuandolosrendimientosacadémicosmediados por el docente fueron más significativos, se podría también aducir como factor explicativo, la habilidad del docente y sus competencias docentes, parallevar estos conocimientos con éxito a los alumnos. 
La aplicación es útil en algunos temas; sin embargo, en otros resultaría como un complemento, ya que los estudiantes obtienen mejores resultados con el docente.

De este estudio, se deduce que existe la necesidad de buscar alternativas para el aprendizaje de la biología y que precisamente el uso de programas de enseñanza asistida por computadores es una opción atractiva, por lo menos como complemento a la clase tradicional, por cuanto permiten que los estudiantes utilicen la aplicación cuando lo juzguen conveniente, repitan los multimedios las veces que sea necesario y estudien a su propio ritmo. Aunque los resultados son alentadores, en algunos casos deberán ser tomados con precaución y adelantar otras investigaciones al respecto.

\section{CONCLUSIONES}

En el rendimiento académico global del subgrupo A, no existe diferencia significativa con un nivel de confiabilidad del $95 \%$. En otros términos, significa que el rendimiento académico de los integrantes de este subgrupo es similar cuando el desarrollo de la clase se hace mediante el uso del aplicativo o la clase tradicional. Esto mismo se puede decir cuando se analizan los resultados del subgrupo B.

El análisis estadístico comparativo del rendimiento académico de los subgrupos $\mathrm{A}$ y $B$ permite concluir que no existe diferencia significativa con un nivel de confiabilidad del $95 \%$, es decir, que cuando se usa el aplicativo multimedial, se obtiene un rendimiento igual al que obtendrían los estudiantes a partir de la clase tradicional.

En los temas lípidos y proteínas hay un efecto positivo del aplicativo, ya que la diferencia entre las medias del rendimiento es estadísticamente significativa.

En el caso de los carbohidratos, el aplicativo no influyó significativamente sobre el rendimiento. Un fenómeno similar ocurrió con el tema bioenergética, en el que los subgrupos rindieron más con la clase tradicional; sin embargo, la diferencia no fue significativa, sugiriendo que, al menos por los datos de esta investigación, el docente es muy importante en la comprensión y en el aprendizaje de dichas temáticas.

Parece ser que el tema enzimas tiene una alta complejidad y dificultad de aprendizaje para los dos subgrupos, puesto que los resultados analizados en términos de las medias de rendimiento, en ambos casos son bajos, incluso inferiores a la nota establecida como aprobatoria.

\section{BIBLIOGRAFÍA}

ALBA P., C. 1998. Perspectivas de futuro en la utilización de las nuevas tecnologías de la información y comunicación en la formación como respuesta a la diversidad. Pixel-Bit. Rev. Medios y Educación. 10:2. Disponible desde Internet en: http://www. sav.us.es/pixelbit/articulos/n10/n10art/art103.htm (con acceso 05/10/06).

ANDRADE G., M.; MIRANDA, J.; FREIXAS S., G. 2001. Predicción del rendimiento académico lingüístico y lógico matemático por medio de las variables modificables de las inteligencias múltiples y del hogar. Rev. Digital de Educación y Nuevas Tecnologías. Contexto Educativo. 3(17):11. Disponible desde Internet en: http://contexto-educativo.com. ar/2001/3/nota-11.htm. (con acceso 17/12/05).

BARTOLOMÉ P., A.R. 1994. Multimedia interactivo y sus posibilidades en educación superior. Pixel-Bit. Rev. Medios y Educación. 1:4. Disponible desde Internet en: http://www.sav.us.es/pixelbit/ (con acceso 17/12/05).

CABERO, J. ; CASTAÑO, C.; CEBREIRO B.; GISBERT. M.; MARTÍNEZ. F.; MORALES. J.; PRENDES. M.; ROMERO R.; SALINAS., J. 2003. Las nuevas tecnologías en la actividad universitaria. Pixel-Bit. Rev. Medios y Educación. 20:13. Disponible desde Internet en: http://www.sav.us.es/pixelbit/articulos/ n20/n20art/art2008.htm (con acceso 20/12/05).

CASTAÑO, G.; CONVERS, J.D.; SOLARTE, I. 2001. Estudio aleatorizado comparativo entre la enseñanza tradicional vs. el uso de computadores e internet, en el curso de Farmacología para estudiantes de pregrado. Universitas Médica. 42(4):9. Disponible desde Internet en: http://med.javeriana.edu.co/publi/vniversitas/serial/v42n4/0041\%20Estudio.PDF. (con acceso 18/11/05)

CONSEJO NACIONAL DE ACREDITACIÓN CNA. 2003. Indicadores para la autoevaluación con fines de acreditación de programas de pregrado en las modalidades a distancia y virtual. (Versión Preliminar). Serie Documentos Especiales, No. 3. Bogotá D.C. 17p. 
EDEL N., R. 2003. El rendimiento académico: concepto, investigación y desarrollo. Rev. Electrónica Iberoamericana sobre Calidad, Eficacia y Cambio en Educación. 1(2):16. Disponible desde Internet en: http://redalyc.uaemex.mx/redalyc/ pdf/551/55110208.pdf (con acceso 15/01/06).

HEALY, D.G.; FLEMING, F.J.; GILHOOLEY, D.; FELLE, P., ALFRED EDWARD WORD, A.E.; GOREY, T., MCDERMOTT, E.W.; FITZPATRICK, J.M.; O'HIGGINS, N.J.; HILL, A.DK. 2005. Electronic learning canfacilitatestudentperformanceinundergraduate surgical education: a prospective observational study. BMC Med. Educ. 5:23. Disponible desde Internet en: http://www.pubmedcentral.nih.gov/articlerender.fcgi?artid=1184079. (con acceso 05/02/08).

HERNÁNDEZ S, R.; FERNÁNDEZ, C.; BAPTISTA L, P. 1997. Metodología de la investigación. México: MC Graw Hill. 162p.

LERNIHAN, O. 2002. An investigation into the potential of the use of multimedia development WebCT to enhance the understanding of information technology for students, within a traditional 3rd level lecturing environment. Tesis de Maestría. Dublin City University, School of Computing. 15p. Disponible desde Internet en: http://www.computing.dcu.ie/research/papers/ MCE/2002/0402.doc (con acceso 10/02/08).

MARRISON, D.L.; FRICK, M.J. 1993. Computer multimedia instruction versus traditional inastruction in post-secondary agricultural education. J. Agr. Education. 34(4):31-38. Disponible desde Internet en: http://pubs.aged.tamu.edu/jae/pdf/Nol34/34-0431.pdf (con acceso 13/02/08).

MOËNNE, G; VERDI, M.; SEPÚLVEDA, E. 2004. Enseñanza de las ciencias con uso de TIC en escuelas urbano marginales de bajo rendimiento escolar. En: Memorias IX Taller Internacional de Software Educativo TISE, Santiago, Chile, Dic. 1,2 y 3 de 2004. p.95-101.
PRESIDENCIA DE LA REPÚBLICA, COLOMBIA. Decreto 2566 de Septiembre de 2003. Artículo 4o.

RECIO S., M.A.; CABERO A., J. 2005. Enfoques de aprendizaje, rendimiento académico y satisfacción de los alumnos en formación en entornos virtuales. Pixel-Bit. Revista de Medios y Educación. 25:93115. Disponible desde internet en: http://www.sav. us.es/pixelbit/articulos/n25/n25art/art2510.htm. (con acceso 14/12/05).

RODRÍGUEZ R., M.J.; PLANCHUELO M., D. 2005. Educación, biblioteca y tic en la sociedad de la información: reto y compromiso. 20p. Disponible desde Internet en: http://www.anabad.org/admin/archivo/ docdow.php?id=158. (con acceso 18/12/05)

SANHUEZA-TSUTSUMI, M.A.; GONZÁLEZ, X.; GUTIÉRREZ, R. 2005. Estudio Exploratorio: Relación de la conciencia corporaly la estructuración témporo-espacial en el aprendizaje de la lecto-escritura en niños de primer año básico. Rev. Iberoamericana de Educación. 35(5):15. Disponible desde internet en: http://www.campus-oei.org/revista/psi_edu22. htm. (con acceso 25/12/05).

SMITH, A.R. Jr..; JONES, J.; CAVANAUGH, C.; VENN, J; WILSON, W. 2006. Effect of Interactive Multimedia on Basic Clinical Psychomotor Skill Performance by Physical Therapist Students. J. Physical Therapy Educ. 11p. Disponible desde internet en: http:// findarticles.com/p/articles/mi_qa3969/is_200610/ ai_n18622053 (con acceso 11/10/07)

UNIVERSIDAD DE CIENCIAS APLICADAS Y AMBIENTALEL. 2005. Proyecto Educativo Institucional. Bogotá D.C.: U.D.C.A. 60p.

Recibido: Noviembre 21 de 2007

Aceptado: Mayo 19 de 2008 